\title{
EL COMPORTAMIENTO EN EL ANÁLISIS DE LAS DECISIONES COLECTIVAS BASADAS EN RAZONES: SESGOS Y EFECTOS ALREDEDOR DEL DILEMA DISCURSIVO
}

\author{
BEHAVIOR IN THE ANALYSIS OF REASON \\ BASED COLLECTIVE DECISIONS: BIASES AND \\ EFFECTS AROUND THE DISCURSIVE DILEMMA
}

\author{
$*$ \\ Gustavo Adrián Bodanza Esteban Freidin \\ Doctor en Filosofía, Profesor Titular Ordinario (UNS) e \\ Investigador Independiente (CONICET). Departamento \\ de Humanidades, Universidad Nacional del Sur e \\ Instituto de Investigaciones Económicas y Sociales del \\ Sur, UNS-CONICET, Argentina. \\ Bahía Blanca, Departamento de Humanidades, \\ Universidad Nacional del Sur, 12 de Octubre y San \\ Juan, 5to piso, tel.: +54 $0291459-5150$ \\ Doctor en Psicología, Investigador Independiente \\ (CONICET). Instituto de Investigaciones Económicas y \\ Sociales del Sur, UNS-CONICET, Argentina. \\ Instituto de Investigaciones Económicas y Sociales del \\ Sur, UNS-CONICET, San Andrés 800, Bahía Blanca, tel.: \\ +5490291 459-5138. \\ efreidindiiess-conicet.gob.ar \\ bodanzadgmail.com; gbodanzadiiess-conicet.gob.ar \\ Este trabajo fue parcialmente financiado por ANPCyT (PICT 2017-1702) y \\ Universidad Nacional del Sur (PGI 24/I223), Argentina.
}

El dilema discursivo es un resultado paradójico que puede suscitarse en las decisiones colectivas, consistente en que la agregación de opiniones de los individuos acerca de una decisión (agregación de conclusiones) puede ser incompatible con la agregación de sus propias opiniones sobre los elementos de juicio relevantes (agregación de premisas). Aunque ha dado lugar a profusas discusiones normativas, pocas investigaciones empíricas se han realizado para estudiar las preferencias de procedimiento de las personas frente al dilema. Aquí hacemos una revisión de los escasos pero iluminadores trabajos experimentales en torno a efectos de marco y sesgos, tales como los de indulgencia, confirmación y la adecuación a normas sociales, que influyen en la elección de un procedimiento en el contexto del dilema discursivo.

Palabras clave: dilema discursivo; decisiones colectivas; normas sociales; sesgo de indulgencia 


\section{6 - Cogency, Journal of reasoning and argumentation}

The discursive dilemma is a paradoxical result that can arise in collective decisions and consists of an incompatibility that may arise from the aggregation of opinions of individuals about a decision (aggregation of conclusions) relative to the aggregation of their opinions on the relevant elements of judgment (aggregation of premises). Although this dilemma has led to profuse normative discussions, few empirical investigations have been conducted to study people's procedural preferences when they face the dilemma. Here we review the scarce but illuminating experimental studies around framing effects and biases, such as indulgence, confirmation, and compliance with social norms, which influence the choice of a procedure in the context of the discursive dilemma.

Keywords: discursive dilemma; collective decisions; social norms; leniency bias 


\section{Introducción}

Kornhauser \& Sager (1993) se refieren a la paradoja doctrinal (doctrinal paradox) como el problema que surge cuando debe llegarse a una decisión para la cual hay dos caminos posibles: sumar los votos de los jurados para el caso global, o bien sumar los votos de los jurados sobre cada uno de los aspectos relevantes y luego combinar los resultados. El problema es que ambos métodos de decisión pueden llevar a decisiones opuestas. La generalización de la paradoja doctrinal a cualquier situación de decisión colectiva de un grupo de agentes a través del voto mayoritario se conoce como dilema discursivo (DD). La Tabla la muestra un contexto de decisión abstracto en el que un jurado formado por tres miembros tiene que tomar una decisión por 'Sİ' o por 'NO' con respecto a una proposición $r$ (la conclusión), que es coimplicada por la conjunción de dos proposiciones $p$ y $q$ (las premisas; i.e., que son necesarias opiniones afirmativas en ambas premisas para sostener una conclusión afirmativa). La opinión de cada jurado sobre $p, q$, y $r$ se muestra en las filas. El dilema surge cuando al aplicar el voto mayoritario sobre los elementos $p$ y $q$ derivando luego la opinión colectiva sobre $r$ (en adelante, procedimiento basado en las premisas -PBP), se obtiene un resultado ('SÍ' en los ejemplos de la Tabla 1) contradictorio con el de aplicar el voto mayoritario sobre la conclusión $r$ directamente ('NO' en los ejemplos de la Tabla 1; en adelante, llamamos a este último procedimiento procedimiento basado en las conclusiones - PBC). La Tabla $1 \mathrm{~b}$ muestra un ejemplo particular de decisión.

\section{Tabla 1}

a. a. Problema general abstracto

\begin{tabular}{|c|c|c|c|}
\hline & P & Q & R \\
\hline Jurado 1 & Sí & NO & NO \\
\hline Jurado 2 & NO & sí & No \\
\hline Jurado 3 & sí & sí & sí \\
\hline Mayoría & sí & sí & sí/NO \\
\hline
\end{tabular}

a. b. Ejemplo específico utilizado en Bodanza et al. (2018) donde debe decidirse si se adjudica un cargo académico a un candidato teniendo en cuenta sus antecedentes docentes y de investigación

\begin{tabular}{|c|c|c|c|}
\hline & $\begin{array}{c}\text { ANTECEDENTES } \\
\text { DOCENTES }\end{array}$ & $\begin{array}{c}\text { ANTECEDENTES DE } \\
\text { INVESTIGACIÓN }\end{array}$ & ¿ADJUDICAR EL CARGO? \\
\hline Jurado 1 & SÍ & NO & NO \\
\hline Jurado 2 & NO & sí & NO \\
\hline Jurado 3 & Sí & sí & Sí/NO \\
\hline Mayoría & sí & sí & \\
\hline
\end{tabular}


Se trata de una vieja paradoja (Condorcet, 1785) que ha dado lugar a la actual teoría de la agregación de juicios (List \& Puppe, 2009). La vasta mayoría de los desarrollos en torno a estos temas ha estado orientada a responder cuáles son las respuestas normativamente más apropiadas a las paradojas u otras situaciones aporéticas y muy poca atención se ha enfocado desde una perspectiva conductual en su análisis. Así lo han notado Bonnefon (2007, 2010), para el caso del DD, y Regenwetter, Grofman, Popova, Messner, Davis-Stober \& Cavagnaro (2007), para la agregación de juicios en general. Nuestro objetivo es realizar un análisis teórico acerca del comportamiento de la gente frente al DD, a partir de los aportes realizados hasta el momento desde una perspectiva empírica experimental en torno a las preferencias de las personas a la hora de elegir entre los dos procedimientos para escapar de la paradoja. Realizaremos esto luego de hacer una revisión de la literatura disponible hasta el momento. Allí rastrearemos los problemas que se puntualizan en las siguientes preguntas: ¿Cómo perciben los individuos la legitimidad de los procedimientos en torno al DD? ¿Influye el enmarque (cómo es descripto el problema) en la elección de un procedimiento? ¿Qué sesgos operan en la elección? De ser identificados ciertos sesgos, ¿qué factores pueden modular sus efectos? ¿Cómo interactúan unos sesgos con otros?

En primer lugar, haremos un repaso no exhaustivo de estudios empíricos previos que resultan relevantes para el problema. En segundo lugar, haremos una revisión exhaustiva de los estudios empíricos realizados en torno al DD. Luego, haremos una revisión de los resultados hallados acerca de sesgos y efectos en la elección de un procedimiento, para luego pasar a un análisis teórico tendiente a brindar explicaciones adecuadas para los resultados vistos.

\section{Antecedentes relevantes}

\section{Marco teórico}

Las investigaciones en torno al juicio de la gente sobre los procedimientos para alcanzar decisiones grupales son relevantes para la comprensión de los factores que pueden modular las percepciones de legitimidad de los procedimientos (Lind \& Tyler, 1988). Thibaut \& Walker (1975) mostraron que las percepciones de la justicia procesal se encuentran entre los determinantes más importantes de las preferencias procesales. Esto puede aplicarse a una variedad de contextos sociales en los que es importante la agregación de votos, decisiones u opiniones, como en organizaciones privadas, partidos políticos o instituciones legales (Tyler, 2006, 2011). Hastie, Penrod \& Pennington (1983) mostraron que los individuos estaban más satisfechos con la decisión del grupo cuando podían deliberar sobre los elementos que apoyaban las decisiones (argumentos o premisas) que cuando deliberaban sólo sobre 
veredictos (conclusiones) individuales. Kameda (1991) mostró que los individuos que deliberaban sobre los elementos (PBP) eran más propensos a aceptar una decisión contraria a su preferencia que los que sólo votaban sobre el veredicto (PBC). A su vez, McCoun \& Kerr (1988) mostraron que las personas tendían a elegir procedimientos que producían resultados indulgentes en jurados simulados, observando un efecto asimétrico en las decisiones dicotómicas: en jurados con divisiones iniciales iguales sobre el veredicto y sin una preferencia individual clara y predominante, la elección estaba más inclinada a la absolución que a la condena. De hecho, algunos autores argumentaron que la tendencia a preferir decisiones que conducen a resultados benevolentes suele ser más fuerte que la preferencia por procedimientos justos (Esaiasson, Persson, Gilljam \& Lindholm, 2016). Estos resultados sugieren que en el caso del DD también pueden esperarse efectos de enmarque, así como el efecto asimétrico de escoger un procedimiento asociado a un resultado benévolo o indulgente. A su vez, surgen preguntas acerca de otras variables que pueden influir en la elección, como la opinión de las personas sobre los resultados de los procedimientos, su participación o no en la decisión y la adecuación a las normas sociales.

\section{Materiales y métodos}

Los estudios experimentales sobre la conducta en torno al DD constituyen una línea de investigación apenas emergente. Con el fin de hallar la literatura enfocada específicamente en tales estudios, el 24 de agosto de 2020 se realizó una búsqueda en la base de datos Scopus, en el campo "Document", sección "Article title, Abstract, Keywords", de las expresiones "discursive dilemma" y "doctrinal paradox", en conjunción con "experiment" y "experimental" (se incluyeron las comillas dobles en las búsquedas). El criterio de búsqueda responde a la necesidad de hallar todos los trabajos en los que se realizaron estudios experimentales en torno al DD. Los resultados fueron, respectivamente, 1 documento (Bodanza, Freidin, Linares \& Delbianco, 2018), 0 documentos, 1 documento (Bonnefon, 2010) y 1 documento (Sekiguchi, 2018). Ante la escasez de resultados, las búsquedas se repitieron en Google Scholar (opción en cualquier idioma), donde se hallaron 269, 263, 89 y 105 documentos, respectivamente. De la lectura de los abstracts de los artículos hallados surgió que sólo reportaban estudios empíricos experimentales en torno al DD los registrados previamente en Scopus, más el trabajo de Bonnefon (2007). También apareció el trabajo de Bonnefon (2011), pero éste sólo presenta una discusión teórica sobre los experimentos reportados por el autor en los trabajos anteriores. En conclusión, podemos afirmar que los primeros trabajos al respecto son los de Bonnefon $(2007,2010)$, los cuales no han tenido impacto en el campo de la psicología social hasta los recientes artículos de Bodanza et al. (2018) y Sekiguchi (2018). A pesar de tratarse de una breve literatura, 
ésta arroja luz sobre importantes efectos e interacciones entre sesgos, a la vez que plantea nuevos problemas para la investigación futura.

\section{Criterios de análisis}

Siguiendo los objetivos del estudio, analizamos en los trabajos señalados la identificación e incidencia de sesgos, su modulación y sus efectos, en la elección entre procedimientos PBP y PBC en torno al DD.

\section{Resultados y discusión}

A continuación, resumimos las estrategias experimentales y los resultados en los trabajos mencionados, para luego pasar al análisis puntualizado de los sesgos y efectos reportados.

Bonnefon (2007) estudia la influencia de tres factores sobre las preferencias entre PBP y PBC: primero, la simplicidad (PBC requiere un solo juicio de cada miembro del grupo de decisión, mientras PBP requiere de un vector de juicios por cada miembro); segundo, en situaciones donde no se aprecia una opinión mayoritaria clara y el resultado puede afectar a un individuo, como en el caso de un juicio penal, es conocido que la preferencia recae sobre el procedimiento que lleva al resultado más benévolo (lenient) (McCoun \& Kerr, 1988), por lo que es de esperar el mismo efecto en el DD; y tercero, si se sabe que las dos condiciones difícilmente pueden satisfacerse simultáneamente, los jueces se sentirán tentados a quedar satisfechos encontrando que se cumpla cada criterio para la mayoría de los jueces, aunque solo una minoría de ellos considere que los dos criterios se satisfacen simultáneamente. Por lo tanto, criterios o condiciones incompatibles deberían alentar la votación mediante PBP. El experimento tuvo un diseño de $2 \times 2$ inter-sujeto. Se les presentó a los participantes $(n=1092)$ una historia en la que un directorio de siete miembros de una compañía debía decidir si aceptar a un candidato en un puesto laboral. Las variables manipuladas fueron la compatibilidad/incompatibilidad entre los criterios (compatibles: joven y trilingüe; incompatibles: joven y experimentado en dirección de equipos) y la polaridad positiva/negativa del enmarque del resultado de la decisión (aceptado/rechazado). Los resultados mostraron que consideraciones de simplicidad favorecieron la preferencia por el PBC, según los propios rankings de simplicidad realizados por los individuos. Segundo, el PBP fue preferido cuando ambas condiciones no eran satisfacibles simultáneamente en la opinión de la mayoría. Tercero, se tendió a favorecer el procedimiento que llevaba al resultado más benévolo. El autor deja planteada una línea de investigación futura sobre la relación entre efectos de polaridad, sesgos de benevolencia o indulgencia y la vasta literatura sobre efectos de enmarque.

Bonnefon (2010) propone una "triple generalización" de su trabajo previo: reforzar la manipulación del enmarque para probar su incidencia en las preferencias; probar también 
su validez en un diseño intra-sujeto; $y$, finalmente, generalizar el resultado para variantes disyuntivas de la paradoja, además de las conjuntivas. En los experimentos, el dilema se presentó en el marco de la evaluación de un empleado de acuerdo con su competencia y motivación para el trabajo. Dos versiones, una conjuntiva $(n=260)$ y una disyuntiva ( $n$ $=244$ ) se combinaron con dos marcos descriptivos, uno positivo (competente, motivado) y uno negativo (incompetente, desmotivado). Los participantes tuvieron que evaluar cada estilo de deliberación y luego responder a la pregunta: ¿Es cierto que el empleado es (in)competente $[y / o]$ está (no está) motivado? Los individuos tenían que evaluar los procedimientos PBP y PBC y elegir uno de los resultados opuestos, mientras el marco variaba entre las condiciones. El primer resultado fue que el comportamiento de los participantes se vio afectado por el marco, incluso en el diseño experimental intra-sujeto. Los individuos tendieron a preferir el procedimiento que conducía a las conclusiones positivas (competente, motivado) y a rechazar el procedimiento que conducía a las negativas (incompetente, desmotivado).

En Bodanza et al. (2018) el problema trató sobre aceptar o rechazar a un candidato para un cargo académico considerando sus méritos docentes y de investigación (tomado de Bovens \& Rabinowicz, 2003, y List, 2006) (Tabla 1b). Considerando los experimentos de Bonnefon, el objetivo fue observar si lo resultados se producían en el nuevo marco y, en caso afirmativo, probar la robustez del sesgo benevolente en interacción con opiniones en sentido contrario. Con 539 participantes, en general, hallaron la misma tendencia indulgente de escoger el procedimiento que llevaba a la aceptación del candidato. Sin embargo, observaron que el efecto era modulado por un sesgo confirmatorio en algunos casos. Más específicamente, la elección entre los procedimientos se emparejaba (diferencias estadísticamente no diferentes al 50\%) cuando coincidía la opinión negativa sobre el candidato (asignada de forma exógena al participante) con rechazar su contratación siguiendo el PBP. Por otra parte, esta modulación no se daba en el caso de coincidencia con el PBC. Esta asimetría se analizará en la subsección Sesgo de confirmación.

En Sekiguchi (2018), el dilema fue descripto como el de la decisión de permitir o prohibir fumar en el lugar de trabajo en consideración del propio interés y del bien común. Las variables independientes fueron la estructura lógica de la agenda (conjuntiva/disyuntiva), el encuadre del objetivo (permitir/prohibir) y la influencia procedimental (qué procedimiento permitía fumar). Como variable de control se consideró la historia como fumadores de los participantes. Los participantes $(\mathrm{N}=1342)$ prefirieron en gran medida el procedimiento que llevaba a la prohibición. Además, los no fumadores fueron intolerantes con el hábito de fumar de otros y tendieron a apoyar los procedimientos conducentes a resultados compatibles con sus experiencias pasadas. Pero además, los resultados mostraron que una opinión minoritaria que no coincidía con la norma social prevaleciente (en este caso, representada 
por el movimiento antitabaco), tuvo más chances de ser apoyada cuando la decisión se tomaba a través del PBC.

A continuación puntualizamos los resultados de acuerdo a los sesgos y efectos en cuestión.

\section{Sesgos y efectos}

\section{Efectos de enmarque}

Los experimentos de Bodanza et al. (2018) y Sekiguchi (2018) corroboraron la sensibilidad a los cambios de marco en torno al DD, con estrategias de diseño de las condiciones similares a las de Bonnefon (2010). Al variar los marcos (elección de un candidato, decisión sobre permitir o prohibir fumar, etc.) se variaban los resultados de las elecciones, cuyas apreciaciones guiaban, en general, la elección del procedimiento. La incidencia de la estructura de la agenda y la interacción de distintos sesgos en tales apreciaciones la analizaremos a continuación.

\section{Estructura lógica de la agenda}

Todos los diseños experimentales sobre el DD han considerado dos estructuras lógicas de la agenda de decisión: conjuntiva y disyuntiva. La estructura conjuntiva (e.g. competente y motivado) permite obtener el DD extrayendo el resultado negativo a través del PBC y el positivo a través del PBP mientras que, inversamente, la estructura disyuntiva (e.g. competente o motivado) lo hace extrayendo el resultado negativo a través del PBP y el positivo a través del PBC (no hay posibilidad lógica de obtener el DD mediante otras combinaciones). Puesto que cada estructura se puede asociar con dos pares del tipo procedimiento-resultado (i.e., conjuntiva: PBP-positivo/PBC-negativo; disyuntiva: PBP-negativo/PBC-positivo) es difícil diferenciar si los posibles efectos obedecen a la estructura o a alguno de los pares procedimiento-resultado asociados. La estrategia de Bonnefon (2010) para resolver este problema consistió en introducir en el experimento dos marcos distintos, uno expresando cualidades positivas (competente, motivado) del empleado sobre el que había que decidir y otro con cualidades negativas (incompetente, desmotivado), de modo que en este último el voto positivo 'Sí acerca de una cualidad implica una opinión negativa acerca del empleado. Esto le permitió ver que el efecto de marco no estuvo dado por la estructura lógica de la agenda sino por el modo en que el resultado sesgaba la elección hacia aquel más benévolo para el empleado.

\section{Sesgo de indulgencia o benevolencia}

El sesgo de benevolencia (beneficiar) o indulgencia (perdonar) consiste en tomar la decisión más beneficiosa para quien pudiera resultar perjudicado o beneficiado con la decisión. Pero 
la benevolencia o no de un resultado no debería de por sí cuestionar lo justo de un procedimiento. Si un procedimiento es percibido como más justo que otro, entonces parece natural que el resultado arrojado por el primero debería resultar más justo que el arrojado por el segundo, independientemente de cuál sea el más benévolo. Sin embargo, los sesgos pueden alterar estos juicios. Los resultados de Bonnefon (2010) constatan, en torno al DD, un efecto similar al de la asimetría hallada por McCoun \& Kerr (1988), que inclina las preferencias sobre los métodos hacia aquel cuyo resultado es más benévolo. Considerando todas las condiciones del experimento, la elección de un procedimiento u otro casi igualaba las chances de una elección aleatoria, con cierta tendencia a elegir PBC en Bonnefon (2007), posiblemente porque el cómputo de PBC es más simple que el de PBP. Sin embargo, los experimentos posteriores de Bonnefon (2010) mostraron un sesgo a elegir aquel procedimiento que arrojaba la aceptación (rechazo) de las conclusiones competente/motivado (incompetente/ desmotivado). En las condiciones conjuntivas la decisión recayó un $61 \%$ sobre el PBP cuando conducía al resultado positivo, mientras que el $45 \%$ eligió ese procedimiento cuando conducía al resultado negativo. Es decir, los participantes se vieron inclinados, en cada marco, a elegir la opción más benévola para el candidato. En las condiciones disyuntivas se observó el mismo sesgo con la elección invertida: 44\% eligió el PBC cuando éste conducía al resultado negativo mientras el 57\% lo eligió cuando conducía al resultado positivo. Para evaluar el acuerdo de los sujetos con las decisiones posibles, Bonnefon (2010) introdujo una escala de 5 puntos, con un rango de -2 (desacuerdo total) a +2 (acuerdo total), para determinar la preferencia por cada procedimiento. En vista de estos resultados, Bodanza et al. (2018) estudiaron la robustez del sesgo de indulgencia, hallando cierta modulación por la interacción de un sesgo de confirmación, como veremos a continuación.

\section{Sesgo de confirmación}

Este sesgo es la tendencia a favorecer, buscar, interpretar y/o recordar la información que confirma las propias opiniones o creencias, otorgando un peso desproporcionadamente menor a posibles alternativas (Plous, 1993, p. 233). Bodanza et al. (2018) pusieron a prueba en qué medida la presencia/ausencia de una opinión proporcionada de forma exógena a los participantes incidía en su elección de procedimiento. El sesgo de confirmación predice que las personas elegirán el procedimiento cuyo resultado coincida con su propia opinión. Por lo tanto, se esperaba que una opinión negativa del candidato modulara el sesgo de indulgencia. Sin embargo, esta predicción no se cumplió en general. Sólo en la condición disyuntiva que mostraba al PBP rechazando al candidato (es decir, cuando 4/6 celdas de las premisas mostraban 'NO' en la Tabla 1) la tendencia indulgente se vio reducida. Diversas explicaciones provistas en la literatura podrían dar cuenta de estos resultados. Bonnefon 
(2010), siguiendo a List (2006), observa que el PBP tiende a evitar falsos positivos (i.e., evita aceptar al candidato cuando no amerita el cargo), mientras el PBC tiende a evitar falsos negativos (i.e., evita rechazarlo cuando lo amerita). Mientras el sesgo indulgente inclinaría la elección hacia el PBC evitando un falso negativo, los sujetos con opinión negativa del candidato podrían encontrar menor temor en rechazarlo sobre una base falsa si las opiniones mayoritarias del jurado sobre los méritos del candidato confirman su misma posición negativa. Siguiendo un argumento de McCoun \& Kerr (1988), si la evidencia de un veredicto particular es clara (como lo sugieren las posiciones negativas convergentes de la mayoría del jurado y del participante), entonces el veredicto favorecido debería ser demostrablemente "correcto" y el sesgo para esa posición debería ser evidente en el esquema de decisión social. Esto sugiere que cuanto más fuerte y clara sea la evidencia contra el candidato, menos pronunciado debería ser el sesgo de indulgencia general. Esto explicaría la disminución en la elección del PBC que produce el resultado positivo. De todos modos, es menester observar que las opiniones puestas a prueba en Bodanza et al. (2018) no fueron espontáneas sino asignadas a los participantes: “Supongamos que en tu opinión, el candidato...". Aunque este procedimiento puede parecer conservador para probar un sesgo de confirmación, el sesgo de confirmación es tan generalizado que se ha observado incluso en condiciones en las que los sujetos no tenían participación material o interés personal evidente (Nickerson, 1998). El problema principal de trabajar con la opinión propia de los participantes es que es difícil que el DD surja espontáneamente en condiciones experimentales, dado que sólo puede darse con determinadas combinaciones lógicas de opiniones. Sin embargo, no es en principio imposible. Una variante del sesgo de confirmación, el sesgo “de mi lado" (my side bias), pareció influir en los experimentos de Sekiguchi (2018). Allí los individuos podían identificarse con un grupo (fumadores/no fumadores) al cual la decisión podía afectar, positiva o negativamente. Como era de esperar, los fumadores tendieron a elegir el procedimiento conducente a permitir fumar. Sin embargo, hubo ciertas diferencias estadísticamente significativas entre un procedimiento y otro, posiblemente como resultado de cómo cada una de las agregaciones hizo manifiesta la norma social representada por el movimiento antitabaco.

\section{Adecuación a las normas sociales}

Los resultados en Sekiguchi (2018) muestran que una opinión minoritaria que no coincide con una norma social prevaleciente (la prohibición de fumar), es más probable que sea sostenida cuando se utiliza el PBC. Sekiguchi explica esto destacando una propiedad básica del PBC: el procedimiento no requiere que los votantes revelen sus razones para llegar a una conclusión. Esta propiedad resultaría atractiva para los participantes que no necesitan exponer sus razones opuestas a la norma. En los experimentos se les pidió a los participantes 
que justificaran su opinión en texto libre. Mediante técnicas de minería de texto, se obtuvo el resultado de que los participantes que apoyaban la prohibición de fumar tendían a usar términos relacionados con la salud ('daño', 'pasivo', 'cuerpo') o con la norma social (e.g., 'tendencia,' 'era', 'sociedad'). Los participantes que apoyaban el permiso para fumar, en cambio, tendían a usar términos relacionados con los procedimientos (e.g., 'regla de mayoría, 'razón', 'derechos', 'minoría,' 'proceso democrático'). Sekiguchi explica que, dado el movimiento generalizado contra el tabaquismo (la norma), los participantes que apoyaron la prohibición de fumar podían justificar fácilmente su opinión, mientras que, contrariamente, los participantes que apoyaron el permiso podían apelar a la imparcialidad del procedimiento evitando referencias a la norma.

\section{Participación en la decisión}

Dado que los participantes en los experimentos de Bonnefon fueron consultados como observadores externos al jurado, Bodanza et al. (2018) consideraron si involucrar a los individuos como miembros del jurado modularía los sesgos de confirmación e indulgencia. La hipótesis fue que tener una voz activa (voto) podía fortalecer el sesgo hacia la opinión proporcionada. De hecho, algunos hallazgos sugieren que las personas perciben que los procedimientos son más justos cuando se les garantiza la expresión de opiniones (Lind \& Tayler, 1988; van den Bos, 2005; Hulst, van den Bos, Akkermans \& Lind, 2017). Por su parte, Thibaut \& Walker (1975) argumentaron que los procedimientos se consideraban más justos cuando los litigantes podían expresar sus preocupaciones en un esfuerzo por influir en el resultado de la decisión. En este sentido, se esperaba que las opiniones proporcionadas ejercieran un efecto más fuerte en las condiciones en las que los participantes tenían un papel de jurado que cuando no lo tenían. Sin embargo, el resultado no arrojó diferencias significativas de ningún tipo, a saber, ni entre las condiciones con opiniones asignadas negativa/positiva, ni entre las condiciones con/sin opinión asignada, ni como modulador del sesgo de indulgencia.

\section{Conclusión}

El estudio de las preferencias de la gente para los procedimientos de decisión grupales o institucionales puede servir para obtener conocimiento sobre los factores predictivos de los juicios de legitimidad, la aceptación de la decisión y la inclinación de la gente a cumplir con las decisiones de las autoridades. No obstante, hemos visto que las preferencias de las personas pueden verse afectadas por factores ajenos a los procedimientos mismos. Los efectos de marco, en particular, el sesgo de benevolencia, pueden inclinar la elección. Incluso teniendo en cuenta que en los trabajos vistos no se probaron las condiciones en las que la elección del 
procedimiento tuvo que hacerse antes de que se conociera ningún resultado, podría darse el caso de que, una vez conocidos, las personas cambien sus preferencias previas. Esta sería una situación relevante que aún debe ser probada.

Otra conclusión interesante se obtiene cruzando los resultados de los estudios experimentales aquí revisados y las observaciones de Dietrich \& List (2007) sobre la manipulación de las decisiones colectivas en torno al DD. Por un lado, los estudios experimentales muestran que, al menos en los marcos en los que la decisión afecta positiva o negativamente a un agente, hay un sesgo general hacia la decisión más benévola o indulgente; esto es, la gente se orienta más hacia la decisión indulgente que a las razones. Por otro lado, el resultado teórico de Dietrich \& List (2007) muestra que cuando los individuos se hayan orientados al resultado de la decisión antes que a las razones, el PBC y el PBP son estratégicamente equivalentes, en el sentido que en ninguno de los dos procedimientos habría incentivos para cambiar estratégicamente el voto con el fin de obtener el resultado deseado. Por lo tanto, si en general los individuos se muestran más preocupados por el resultado entonces, teóricamente, no habría diferencias entre un procedimiento y otro en lo que respecta a la manipulación de la decisión.

Entre los problemas metodológicos que se observan en los experimentos, una limitación importante es la de probar escenarios hipotéticos, jurados simulados y opiniones asignadas a los participantes. La necesidad de trabajar bajo estas limitaciones obedece principalmente a que la probabilidad de que el DD surja espontáneamente es aparentemente baja. Asignar a un grupo la tarea de tomar una decisión y esperar a que el dilema surja de las opiniones subjetivas y los veredictos de los participantes resulta inverosímil. No obstante, como una solución parcial puede ser importante incluir más realismo en estudios futuros, como, por ejemplo, mediante la implementación de incentivos económicos asociados a las decisiones.

\section{Agradecimientos}

Agradecemos los comentarios de un/a revisor/a que ayudaron a mejorar la legibilidad del artículo. Este trabajo fue parcialmente financiado por ANPCyT (PICT 2017-1702) y Universidad Nacional del Sur (PGI 24/I223), Argentina.

\section{Referencias}

Bodanza, G., Freidin, E., Linares, S., \& Delbianco, F. (2020). Modulation of the leniency bias in the discursive dilemma. International Journal of Psychology, 55(1), 67-75. https://doi.org/10.1002/ijop.12545 
Bodanza, Gustavo, Esteban Freidin, Sebastián Linares, \& Fernando Delbianco (2018). Modulation of the leniency bias in the discursive dilemma. International Journal of Psychology, pre-published. https://doi.org/10.1002/ijop.12545

Bovens, L., \& Rabinowicz, W. (2004). Democracy and argument: Tracking truth in complex social decisions. En A. van Aaken, C. List, \& C. Luetge (eds.), Deliberation and Decision: Economics, Constitutional Theory, and Deliberative Democracy (pp. 143-157). Ashgate Publishing. http://www.ashgate.com/Bovens, Luc \& Wlodek Rabinowicz (2003). Democracy and argument - Tracking truth in complex social decisions, in A. van Aaken, C. List \& C. Luetge (eds.), Deliberation and Decision, Aldershot (Ashgate Publishing).

Bovens, L., \& Rabinowicz, W. (2006). Democratic answers to complex questions - An epistemic perspective. Synthese, 150(1), 131-153. https://doi.org/10.1007/s11229$\underline{006-0005-1}$

Bovens, Luc, \& Wlodek Rabinowicz (2006). Democratic answers to complex questions - An epistemic perspective. Synthese 150(1): 131-153. https://doi.org/10.1007/ s11229-006-0005-1

Bonnefon, J.-F. (2007). How do individuals solve the doctrinal paradox in collective decisions?: An empirical investigation. Psychological Science, 18(9), 753-755. https:// doi.org/10.1111/j.1467-9280.2007.01972.x

Bonnefon, Jean-Francois (2007). How Do Individuals Solve the Doctrinal Paradox in Collective Decisions?. Psychological Science, 18(9), 753-755. http://dx.doi. org/10.1111/j.1467-9280.2007.01972.x

Bonnefon, J.-F. (2010). Behavioral evidence for framing effects in the resolution of the doctrinal paradox. Social Choice and Welfare, 34(4), 631-641. https://doi.org/10.1007/ $\underline{\text { s00355-009-0421-5 }}$

Bonnefon, Jean-Francois (2010). Behavioral evidence for framing effects in the resolution of the doctrinal paradox. Social Choice And Welfare, 34(4), 631-641. https://doi. org/10.1007/s00355-009-0421-5

Bonnefon, J.-F. (2011). The doctrinal paradox, a new challenge for behavioral psychologists. Advances in Psychological Science, 19(5), 617. 
Bonnefon, Jean-Francois (2011). The doctrinal paradox, a new challenge for behavioral psychologists. Advances in Psychological Science, 19(5), 617-623. https://doi. org/10.3724/SP.J.1042.2011.00617

Brennan, G. (2001). Collective coherence?. International Review of Law and Economics, 21(2), 197-211. https://doi.org/10.1016/S0144-8188(01)00056-4

Brennan, Geoffrey (2001). Collective coherence? International Review of Law and Economics 21(2), 197-211. https://doi.org/10.1016/S0144-8188(01)00056-4

Condorcet, J.-A.-N. de C. (1785). Essai sur l'application de l'analyse à la probabilité des décisions rendues à la pluralité des voix ([Reprod.]) / par M. le marquis de Condorcet. https://gallica.bnf.fr/ark:/12148/bpt6k417181

Condorcet, Marie-Jean-Antoine Nicolas de Caritat (1785). Essai sur l'application de l'analyse à la probabilité des décisions rendues à la pluralité des voix, l'Imprimerie Royale, Paris.

Dietrich, F. (2006). Judgment aggregation: (Im)possibility theorems. Journal of Economic Theory, 126(1), 286-298. https://doi.org/10.1016/j.jet.2004.10.002 Dietrich, Friedrich. (2006). Judgment aggregation: (im)possibility theorems. Journal of Economic Theory, 126(1), 286-298. https://doi.org/10.1016/j.jet.2004.10.002

Dietrich, F., \& List, C. (2007). Strategy-proof judgment aggregation. Economics \& Philosophy, 23(3), 269-300. https://doi.org/10.1017/S0266267107001496

Dietrich, Friedrich \& Christian List (2007). Strategy-proof judgment aggregation, Economics and Philosophy, 23(03), 269-300. https://doi.org/10.1017/S0266267107001496

Esaiasson, P., Persson, M., Gilljam, M., \& Lindholm, T. (2019). Reconsidering the role of procedures for decision acceptance. British Journal of Political Science, 49(1), 291314. https://doi.org/10.1017/S0007123416000508

Esaiasson, Peter; Mikael Persson; Mikael Gilljam \& Torun Lindholm (2016). Reconsidering the role of procedures for decision acceptance. British Journal of Political Science, 1-24. https://doi.org/10.1017/S0007123416000508

Hastie, RaidR.,; Steven Penrod, S. \& Nancy Pennington, N. (1983) Inside the jJury. Cambridge, MA: Harvard University Press. 
Hulst, LiesbethL.,; Kees van den Bos, K.,; Arno Akkermans, A. \& Allan Lind, A. (2017). On the psychology of perceived procedural justice: experimental evidence that behavioral inhibition strengthens reactions to voice and no-voice procedures. Frontiers in Psychological and Behavioral Science, 6(1), 1-12.

Kameda, Tatsuya T. (1991). Procedural influence in small-group decision making: deliberation style and assigned decision rule. Journal of Personality and Social Psychology, 61(2), 245-256. http://dx.doi.org/10.1037/0022-3514.61.2.245

Kornhauser, Lewis L. \& Lawrence Sager, L. (1993). The one and the many: adjudication in collegial courts. California Law Review, 91, 1-51. https://doi.org/10.15779/ $\underline{\mathrm{Z} 38 \mathrm{C} 43 \mathrm{~K}}$

Lind, E. A., \& Tyler, T. R. (1988). The social psychology of procedural justice. Springer US. https://doi.org/10.1007/978-1-4899-2115-4

Lind, E.Allan \& Tom Tyler (1988). The social psychology of procedural justice. Springer Science+Business Media, LLC.

List, Christian C. (2006). The discursive dilemma and public reason. Ethics, 116(2), 362402. https://doi.org/10.1086/498466

List, C., \& Puppe, C. (2009). Judgment aggregation: A survey. En P. Anand, P. Pattanaik, \& C. Puppe (eds.), Handbook of Rational and Social Choice (pp. 457-482). Oxford University PressList, Christian \& Clemens Puppe (2009). Judgment aggregation: A survey. In The Handbook of Rational and Social Choice, ed. by P. Anand, P. Pattanaik \& C. Puppe, Oxford University Press, Chapter 19.

Nickerson, Raymond R. (1998). Confirmation bias: A ubiquitous phenomenon in many guises. Review of General Psychology, 2(2), 175-220. http://dx.doi.org/10.1037/1089$\underline{2680.2 .2 .175}$

Plous, Scott S. (1993). The psychology of judgment and decision making, McGraw-Hill, ISBN 978-0-07-050477-6, OCLC 26931106

Regenwetter, MichelM., ; Bernard Grofman, B., ; Anna Popova, A.; William Messner, W, ; Clintin Davis-Stober, C. \& Daniel Cavagnaro, D. (2009). Behavioural social choice: a status report. Philosophical Transactions of the Royal Society B: Biological Sciences, 364(1518), 833-843. http://doi.org/10.1098/rstb.2008.0259 
Sekiguchi, T. (2019). Preferences over procedures and outcomes in judgment aggregation: An experimental study. Theory and Decision, 86(2), 239-258. https://doi. org/10.1007/s11238-018-9678-4

Sekiguchi, Takuya (2018). Preferences over procedures and outcomes in judgment aggregation: an experimental study. Theory and Decision. https://doi.org/10.1007/ s11238-018-9678-4

Thibaut, John J. \& Laurens Walker, L. (1975). Procedural justice: a psychological analysis. Lawrence Erlbaum Associates, Hillsdale.

Tyler, T.om (2006). Why people obey the law. Princeton University Press.

Tyler, T.om (2011). Why people cooperate. The role of social motivations. Princeton University Press.

Van den Bos, K.ees (2005). What is responsible for the fair process effect? EIn Greenberg, J. and J. Colquitt, J. (eds.) Handbook of Organizational Justice (pp. , ed. by J. Greenberg and J. Colquitt, 273-300). Mahwah, NJ: Lawrence Erlbaum Associations. 